\title{
A Homeostasis Hypothesis of Avian Influenza Resistance in Chickens
}

\author{
Jing An ${ }^{1,2}{ }^{-}$, Jinxiu $\mathrm{Li}^{2,+}$, Ying Wang ${ }^{3}$, Jing Wang ${ }^{2}$, Qinghe $\mathrm{Li}^{2}$, Huaijun Zhou ${ }^{3}{ }^{\oplus}$, \\ Xiaoxiang $\mathrm{Hu}^{2,+, *}$, Yiqiang Zhao ${ }^{1,2, t, *}$ and $\mathrm{Ning} \mathrm{Li}^{2}$ \\ 1 Beijing Advanced Innovation Center for Food Nutrition and Human Health, College of Biological Sciences, \\ China Agricultural University, Beijing 100193, China \\ 2 State Key Laboratory of Agrobiotechnology, College of Biological Sciences, China Agricultural University, \\ Beijing 100193, China \\ 3 Department of Animal Science, University of California, Davis, CA 95616, USA \\ * Correspondence: huxx@cau.edu.cn (X.H.); yiqiangz@cau.edu.cn (Y.Z.) \\ + These authors contributed equally to this work.
}

Received: 8 May 2019; Accepted: 11 July 2019; Published: 17 July 2019

\begin{abstract}
Avian influenza has caused significant damage to the poultry industry globally. Consequently, efforts have been made to elucidate the disease mechanisms as well as the mechanisms of disease resistance. Here, by investigating two chicken breeds with distinct responses to avian influenza virus (AIV), Leghorn GB2 and Fayoumi M43, we compared their genome, methylation, and transcriptome differences. MX1, HSP90AB1, and HSP90B1 exhibited high degrees of genetic differentiation $\left(\mathrm{F}_{\mathrm{ST}}\right)$ between the two species. Except for the $M X 1$-involved direct anti-virus mechanism, we found that at the methylation and transcriptome levels, the more AIV-resistant breed, Fayoumi, exhibited less variation compared with Leghorn after AIV inoculation, which included change trends in differentially expressed regions, top-fold change genes with FDR-corrected $p<0.05$, immune response related genes, and housekeeping genes. Fayoumi also showed better consistency regarding changes in methylation and changes at the transcriptome level. Our results suggest a homeostasis hypothesis for avian influenza resistance, with Fayoumi maintaining superior homeostasis at both the epigenetic and gene expression levels. Three candidate genes-MX1, HSP90AB1, and HSP90B1—showed genetic differentiation and altered gene expression, methylation, and protein expression, which merit attention in further functional studies.
\end{abstract}

Keywords: Fayoumi; Leghorn; difference degrees; fold change levels; methylation; FST; RNA-seq

\section{Introduction}

Avian influenza is a highly contagious disease. The disease is caused by avian influenza virus (AIV), which is classified as belonging to the Orthomyxoviridae family [1]. Avian influenza virus has not only caused great economic damage, but also poses a serious threat to human lives [2]. Numerous AIV vaccines have been developed to prevent its spread in chickens or from chickens to humans. However, due to the rapid mutation of the pathogens and time taken to develop effective vaccines, AIV has not been under complete control. Consequently, understanding the mechanisms of AIV inoculation and resistance is urgent to better control avian influenza outbreaks.

Different bird species exhibit distinct immunologic responses towards AIV infections [3]. It is reported that $M X 1$ is relevant to reductions in morbidity, viral shedding, and cytokine responses in chickens with highly pathogenic AIV inoculation [4,5]. Researchers created genetically modified chickens, which overexpressed a small hairpin RNA with a chicken U6 promoter that regulated viral RNA polymerase, making them unable to transmit AIV to other chickens [6]. Transgenic chickens 
expressing the 3D8 single chain variable fragment protein have also been generated, which was demonstrated to suppress avian influenza transmission [7].

Due to its superior reproduction trait, the Leghorn chicken plays an important role in the commercial egg supply market [8]. Unfortunately, however, it has very little ability to resist diseases, including avian influenza. Fayoumi is an indigenous chicken breed that originated in Egypt. It is well known for its strong resistance to various pathogens, and it is very robust to extreme environmental challenges $[9,10]$. To discover the underlying mechanisms of distinct phenotypic differences in avian influenza resistance, Leghorn GB2 and Fayoumi M43 have previously been compared at the DNA, expression, and methylation levels [9,11-13]. Wang et al. identified differentially expressed genes before and after the avian influenza virus inoculation and reported novel signaling pathways associated with disease resistance, including oxygen transport, gas transport, and the establishment of localization [12]. Recently, Li et al. compared differences in the whole genome methylation pattern between the two breeds [11]. However, the methylation data were collected under normal conditions only for the two breeds, and no AIV inoculation experiments were performed. It is worth noting that disease resistance does not mean free from infection. A previous study showed a considerable amount of virus titers in infected Fayoumi, even though they appeared less clinically ill than the Leghorn chickens [12]. This suggests that they have an alternative approach to disease resistance, whereby they have better survival rates after contracting the virus, in addition to having a direct anti-virus mechanism.

Homeostasis is defined as the ability to maintain a stable internal environment in the presence of disturbances [14], which has been observed in numerous biological processes [11,15-19], such as oxidative stress and calcium metabolism [20]. After AIV invades the hosts, besides activating anti-virus procedures including the NF- $\mathrm{KB}$ signaling pathway or the RIG-I signaling pathway, it is also important to protect hosts from tissue lesions that result from immune over-reaction, i.e., surviving with reduced body damage. This is consistent with the concept of canalization that was originally described by Waddington [21], which predicts the ability to maintain a constant phenotype regardless of environmental perturbations [22]. In this case, AIV is regarded as the environmental perturbation.

In this study, we extended our previous work on the DNA methylation comparison between Leghorn and Fayoumi chickens [11]. We investigated differentially methylated regions as well as top-fold change genes with FDR-corrected $p<0.05$, between individuals with AIV (avian influenza $\mathrm{H} 5 \mathrm{~N} 3$, low pathogenicity avian influenza, LPAI) inoculation compared to individuals challenged with PBS in both breeds. We found that for both methylation and transcriptome levels, the more AIV-resistant breed, Fayoumi, exhibited less variation compared to Leghorn chickens after AIV inoculation. Based on our results, we proposed a novel hypothesis for an AIV resistance mechanism, which could be used as a supplement to traditional anti-virus mechanisms that focus on destroying or blocking the virus. Namely, Fayoumi can better maintain internal homeostasis in both methylation and gene expression levels to avoid over-reactions due to AIV infections.

\section{Materials and Methods}

\subsection{Samples and Experimental Design}

All experiments were conducted in two genetically distinct and highly inbred chicken lines, Leghorn GB2 and Fayoumi M43, which have an inbreeding coefficient of more than $99.9 \%$ and the specific experimental designs has been listed as follows. The samples and replicates have been artificially selected by the Iowa State group since 1954 [13]. Blood DNA samples from 16 individuals per line were pooled for resequencing [13]. Whole genome wide methylome samples were generated from eight lung tissue libraries for each line, which were inoculated with avian influenza H5N3 (LPAI) or PBS (Phosphate Buffer Saline), and lung samples were harvested at $96 \mathrm{~h}$ post-inoculation. RNA sequencing samples were generated from the same experimental design and generated from Wang et al. [12]. All animal experiments were conducted in our collaborative laboratory at the Texas A\&M University, and data has been published previously [12]. 


\subsection{RNA-seq Data Analysis}

The samples used for RNA-seq data analysis were taken from Wang et al. [12]. Eight three-week-old chickens were randomly chosen from individuals challenged with a $10^{7} 50 \%$ egg infective dose of H5N3 AIV (LPAI) or PBS. There were two birds per breed per treatment. Based on a previous test, which showed that lesion severity in chicken lungs hit its peak at four days post-inoculation, the total RNA of the lungs and trachea was harvested and extracted by the trizol method in accordance with the manufacturer's instructions [12]. Reads with adapters and low Phred qualities were also removed by the FASTX-Toolkit (http://hannonlab.cshl.edu/fastx-toolkit). Qualified reads were mapped against the reference genome (GRCg6a) using the Tophat software [23] with default parameters. Bam files were utilized to assemble transcripts using cufflinks [23]. Top-fold change genes with FDR-corrected $p<0.05$ were identified by cuffdiff [23]. The gene ontology (GO) term annotation was conducted using the bioconductor topGO package, which used Fisher's exact test to perform enrichment tests of top-fold change genes. In addition, to reveal the enrichment degrees of relevant pathways in both lines, bubble plots were plotted using the ggplot2 package, and these are presented in Figures S1 and S2.

Top-fold change genes were calculated using cuffdiff, with the level of significance set as an FDR-corrected $p$-value below 0.05 . The gene expression difference and fold change levels of the top-fold change gene set, the immune response related gene set, the housekeeping gene set, and the gene set as a whole were calculated as follows: (1) Fayoumi gene expression difference degrees were measured as the mean values of $\log _{2}$ (Fayoumi gene expression values with AIV inoculation/Fayoumi gene expression values challenged with PBS) $\left(\log _{2}(\right.$ FA_G/FP_G) $)$ and Fayoumi gene expression fold change levels were measured as the variance of $\log _{2}$ (FA_G/FP_G); (2) Leghorn gene expression difference degrees were measured as the mean value of $\log _{2}$ (Leghorn gene expression values with AIV inoculation/Leghorn gene expression values challenged with PBS) $\left(\log _{2}\left(\mathrm{LA} \_\mathrm{G} / \mathrm{LP} \_\mathrm{G}\right)\right)$ and Leghorn gene expression fold change levels were measured as the variance of $\log _{2}($ LA_G/LP_G); (3) a comparison of gene difference between Fayoumi and Leghorn was conducted using t-tests and a comparison of gene expression fold change levels between Fayoumi and Leghorn was conducted using F-tests.

\subsection{Whole Genome Bisulfite Sequencing Data Analysis}

DNA samples of lung tissue were collected at $96 \mathrm{~h}$ post-inoculation with avian influenza H5N3 (LPAI) or PBS, respectively. These DNA samples were extracted according to the phenol chloroform isoamylalcohol method. After DNA was sonicated to 200-400 bp, an end repair job was completed with a triphosphate mix free of dCTP. The following ligation procedures, methylation adapters, and DNA library sequencing were conducted on the Illumina sequencing platform. Raw whole genome methylation sequencing data were generated from the Illumina Genome Analyzer II. The processed reads were then converted and mapped against the reference genome (GRCg6a) using the Bismark software [24], which was also used to quantify the methylation ratios of single sites in the chicken genome. Differentially methylated sites and differentially methylated regions were calculated by Radmeth [25] under the beta-binomial regression model with an FDR-corrected $p$-value below 0.01 .

The DMRs locations were annotated with the reference annotation file (GRCg6a gtf). The gene body was defined as the region from the start codon to the stop codon. The promoter region was defined as $5 \mathrm{~kb}$ upstream of the start codon, while the upstream region was defined as $5 \mathrm{~kb}$ upstream of the promoter, and the distant region was defined as $5 \mathrm{~kb}$ upstream of the upstream region. The methylation level of each gene was obtained by averaging the methylation ratios of individual sites in the gene body. Since four replicates were inoculated with AIV or challenged with PBS in both Fayoumi and Leghorn chickens, $\log _{2}$ (gene methylation ratios of samples with AIV/gene methylation ratios of samples with PBS) were calculated for each individual gene or particular gene set to determine the methylation difference as well as the fold change level. Again, t-tests were used to compare the gene methylation difference, and F-tests were used to compare gene methylation fold change levels between Fayoumi and Leghorn chickens. 


\subsection{Resequencing Data Analysis}

Genomic resequencing data from the Fayoumi and Leghorn breeds were retrieved from Fleming et al. [13]. Raw reads were mapped against the reference genome (GRCg6a Ensemble database version) using BWA (Burrows-Wheeler Alignment tool) [26] with default settings. The Genome Analysis Toolkit (GATK) 3.2.2 [27] was utilized for SNP calling for each group following the GATK best practice pipeline. The fixation index $\left(\mathrm{F}_{\mathrm{ST}}\right)$ values at single nucleotide resolution were calculated according to the Weir and Cocker-ham (1984) formula [28] using in-house Perl scripts. By employing a sliding window approach with a window size of $10 \mathrm{Kbp}$ and a step size of $10 \mathrm{Kbp}$, the genetic differentiation between Fayoumi and Leghorn was averaged for each window throughout the whole genome. Similarly, to quantify genetic differentiation at the gene level, $\mathrm{F}_{\mathrm{ST}}$ values for individual genes were obtained by averaging single nucleotide $\mathrm{F}_{\mathrm{ST}}$ values from the start codon to the stop codon of a gene.

\section{5. $d n / d s$ Ratio Calculation}

To calculate the $\mathrm{dn} / \mathrm{ds}$ ratio (the ratio of the number of synonymous substitutions per synonymous site to the number of nonsynonymous substitutions per nonsynonymous site) for MX1, HSP90AB1, and HSP90B1, we first extracted CDS sequences for the reference genome, as well as for Fayoumi and Leghorn, using the bedtools getfasta function [29]. Then, fasta sequences were translated into amino acids with the HyPhy 2.2.4 version data file tools [30]. The $\mathrm{dn} / \mathrm{ds}$ ratios of the three genes were measured on the Datamonkey 2.0 server [31], which was based on the mixed effects branch-site model of the MEME software [32].

\subsection{Data Availability}

Our sequencing data have already been submitted to the NCBI Gene Expression Omnibus (http://www.ncbi.nlm.nih.gov/geo) under accession nos. GSE56975 and GSE128053.

\section{Results}

\subsection{Less Gene Expression Fold Change Levels in Response to Avian Influenza Virus in Fayoumi}

Since our RNA-seq data were generated from Wang et al. [12], the phenotype differences in AIV resistance between Fayoumi and Leghorn have already been tested. There were two birds per breed per treatment. Infected chicken lungs hit the peak at four days post-inoculation, and virus titers in infected Fayoumi $5.270 \log _{10} \mathrm{EID}_{50} \mathrm{~mL}^{-1}\left(\mathrm{EID}_{50}=50 \%\right.$ egg infective dose) were lower than those in Leghorn chickens $\left(6.480 \log _{10} \mathrm{EID}_{50} \mathrm{~mL}^{-1}\right)$. The lesion scores for Fayoumi and Leghorn chickens were $55 \%$ and $80 \%$, respectively [12], which indicated that Fayoumi chickens can block viral entry/replication to some degree and were healthier compared to Leghorn chickens after AIV inoculation. To explore the altered expression patterns in both breeds, we re-analyzed the RNA-sequencing data.

The results showed that after being inoculated with AIV, many genes exhibited altered expression patterns. Thirty-five differentially expressed genes ( $p<0.05$ adjusted by FDR) were mainly enriched in the pathways of respiratory burst and mitochondrial calcium ion homeostasis between Fayoumi individuals with AIV inoculation (FA) compared to Fayoumi individuals challenged with PBS (FP) FA compared to FP (Figure S1). Fifty differentially expressed genes $(p<0.05$ adjusted by FDR) were mainly enriched in the pathways of cell metabolism and response to xenobiotic stimulus between Leghorn individuals with AIV inoculation (LA) compared to Leghorn individuals challenged with PBS (LP) (Figure S2). For both Leghorn and Fayoumi chickens, differentially expressed genes were enriched in function terms related to the immune response (Figures S1 and S2).

For the top-fold change gene set, the fold change or difference between expression levels after inoculation with AIV was calculated for both Leghorn and Fayoumi (see Materials and Methods). The comparison using t-tests showed that the difference between expression levels of Fayoumi was significantly smaller than those of Leghorn chickens, with a $p$-value of 0.026 . As shown in Figure 1A, the F-test results revealed that the gene expression fold change levels of top-fold change genes in Fayoumi 
were also significantly smaller than those in Leghorn chickens with a $p$-value of 0.049 . This suggests that Fayoumi can better maintain internal homeostasis following exposure to an external stimulus, e.g., AIV inoculation, while Leghorn chickens are more susceptible to the pathogen and have more difficulty stabilizing gene expression.

A

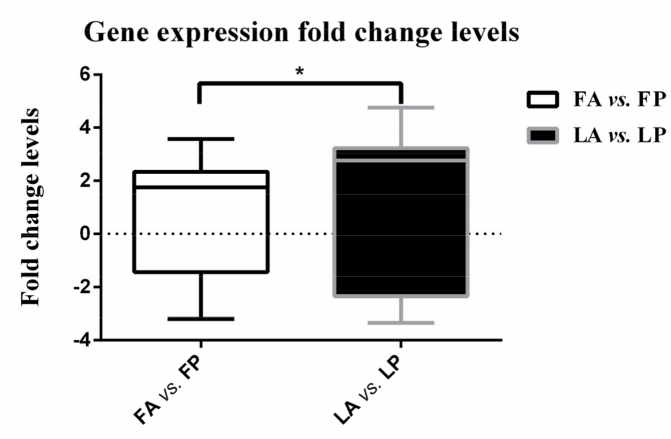

B

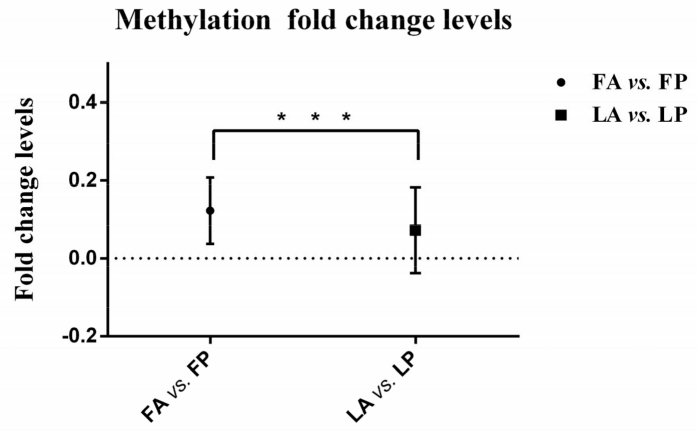

Figure 1. Gene and methylation expression fold change levels in Fayoumi and Leghorn breeds. (A) Gene expression fold change levels of top-fold change genes with false discovery rate (FDR)-corrected $p<0.05$, which were measured as the variance of $\log _{2}$ (gene expression levels of individuals with avian influenza virus (AIV) inoculation/gene expression levels of individuals challenged with phosphate buffer saline (PBS)). The comparison used F-tests, the y-axis represents mean with SD (Standard Deviation), and * indicates a significance level of 0.05 ; (B) methylation fold change levels of differentially methylated regions, which were measured as the variance of $\log _{2}$ (methylation ratios of individuals with AIV inoculation/methylation ratios of individuals challenged with PBS). The comparison used F-tests, the $\mathrm{y}$-axis represents the mean with $\mathrm{SD}$, and ${ }^{* * *}$ indicates a significance level of 0.01 .

For immune response related genes (genes listed in Table S1), by comparing $\log _{2}$ (FA_G/FP_G) and $\log _{2}$ (LA_G/LP_G), more genes were observed to be upregulated in innate immune responses in Leghorn chickens, whereas more genes were upregulated in the adaptive immune response in Fayoumi chickens (as shown in Table S1). In this case, the immune response may constitute a double-edged sword, in which it can both inhibit replications of AIV and damage the organism itself. We also noticed fold changes of genes involved in tissue lesions, such as those involved in the oxidative stress pathway, the apoptosis pathway, and the ferroptosis pathway, were much smaller in Fayoumi compared with Leghorn chickens (Table S1). Interestingly, it was found that the gene expression change degrees of the homeostasis indicator HSP90AB1 [33] were upregulated more in Fayoumi compared to in Leghorn chickens $\left(\log _{2}\left(\right.\right.$ FA_G/FP_G) $/ \log _{2}($ LA_G/LP_G $\left.)\right)=6.0851$, Table S1). As a supplement to traditional anti-virus mechanisms that focus on destroying or blocking the virus, Fayoumi chickens may be able to better maintain internal homeostasis in gene expression levels allowing them to avoid over-reactions due to AIV infections.

\subsection{Avian Influenza Virus Inoculation Introduced Changes in DNA Methylation in Both Breeds}

To reflect dynamic responses to an external stimulus, we performed whole genome bisulfite sequencing (WGBS) on the lung tissues of two three-week-old chickens (Fayoumi and Leghorn) that were either inoculated with $10^{7} 50 \%$ egg infective dose (EID $\mathrm{E}_{50}$ ) of low-path H5N3 AIV or challenged with PBS. For each individual, we generated two technical replicates. In total, 16 WGBS libraries were generated (two breeds $\times$ two conditions $\times$ two individuals $\times$ two replicates) (detailed in Materials and Methods). Filtered clean reads from the 16 WGBS sequencing data ranged from 154 million to 516 million per sample (Table S2). The mapping rates were about $86.2 \%$ (Table S2).

The majority of CpG sites (60 80\%) in the genome were methylated, which is consistent with a previous study [34]. As shown in Figure S3, we plotted the average DNA methylation level of all 16 samples proportionately along the gene structure. To reveal the difference in the methylation pattern, 
we focused on two comparisons: FA compared to FP and LA compared to LP. For each comparison, analyses of differentially methylated sites (DMSs) and differentially methylated regions (DMRs) were performed using the Radmeth software [25]. As listed in Supplementary Table S3, we identified 2143 DMSs from the FA compared to FP comparison and 162 DMSs from the LA compared to LP comparison. It is well known that DNA methylation functions differently depending on its location [35]. Methylation in the gene body has been reported to be relevant to the process of alternative splicing [36], while methylation in promoters is associated with transcriptional activation [35]. To explore the distribution features of DMRs, we scanned the locations of DMRs for both breeds (FA compared to FP and LA compared to LP). In the gene region, $10.5 \%$ of DMRs were located in the gene body, and the rest were annotated at the promoter and distant regions ( $>10 \mathrm{~kb}$ ). Within the gene body, 29.4\% of DMRs were located at the lncRNA (long non-coding RNA) genes. To test whether the differences in methylation sites or degrees between the two breeds were due to unequal efficiency of methylation-related enzymes, we checked the changes in gene expression for methylation-related enzymes. For FA compared to FP and for LA compared to LP, DNMT1 was downregulated with fold changes of $0.9215(p=0.8663)$ and 0.6433 ( $p=0.4283)$, respectively. For DNMT3a, the fold change in FA compared to FP was 1.0605 with $p=0.7953$, while for the Leghorn group, the fold change was 1.5638 with $p=0.1983$. For DNMT3b, the corresponding values were 0.5433 and 1.0378 for Fayoumi and Leghorn chickens, respectively $(p>0.05)$.

\subsection{Less DNA Methylation Fold Change Levels in Response to Avian Influenza Virus in Fayoumi}

In addition to identifying DMSs and DMRs, the fold changes or differences in methylation levels of DMRs were also measured (see Materials and Methods). The comparison using t-tests showed that the Fayoumi methylation difference level was much smaller than those of Leghorn chickens with $p<0.0001$. As shown in Figure 1B, the methylation fold change levels in Fayoumi chickens were also significantly smaller than those in Leghorn chickens (F-test, $p<0.0001$ ), which again supported the hypothesis that Fayoumi chickens can better maintain internal homeostasis. To explore the functional roles of DMRs in Fayoumi and Leghorn chickens, we annotated genes located at DMRs, as listed in Table S4. We found that multiple genes were enriched in immune response related pathways.

We separately addressed the methylation status of immune response related genes (the same gene lists as in Table S1: Innate immune response, adaptive immune response, and oxidative stress related pathways). To retrieve the gene level methylation ratios, we first averaged methylation ratios of each site along a gene from the start codon to the stop codon. For all immune response related genes, we calculated the methylation change degree. We found it was smaller in Fayoumi compared to Leghorn chickens, although it was not shown to be statistically significant using t-tests (Table S5). However, as shown in Figure 2, the gene methylation fold change degree of most immune response related genes was significantly smaller in Fayoumi than in Leghorn chickens. This suggests that Fayoumi chickens have a greater ability to avoid an excessive immune response. 
A Methylation fold change levels of innate immune response relited genes

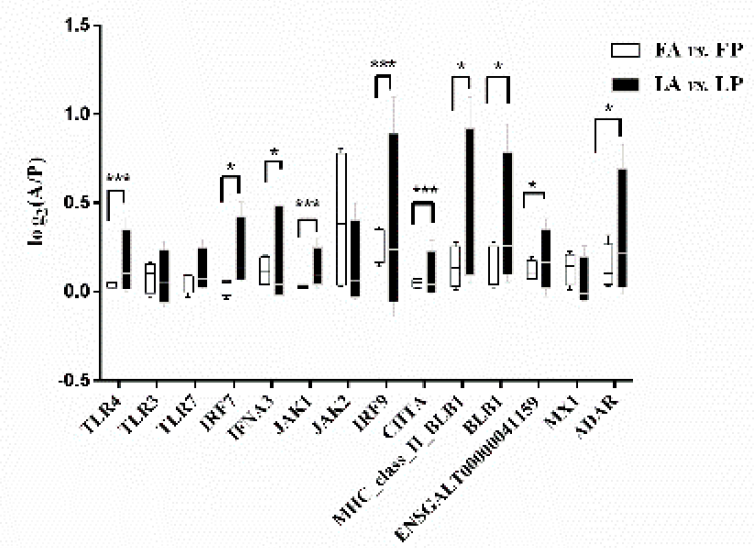

B Methylation fold change levels of adaptive immune response related genes

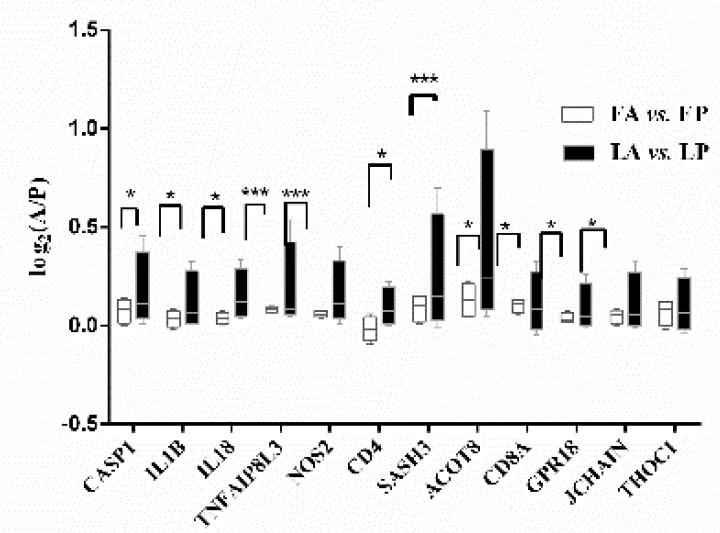

C. Methylation fold change levels of oxidative stress related genes

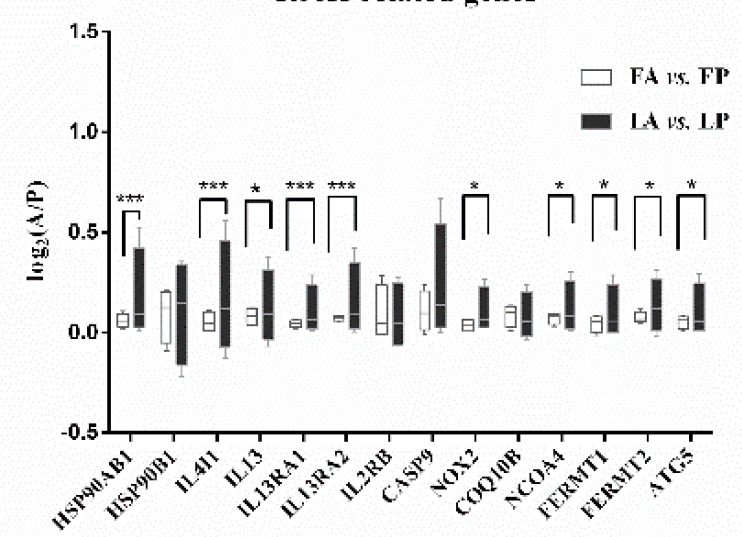

Figure 2. Gene methylation fold change levels of immune response related genes. The variance of $\log _{2}$ (gene methylation ratios of individuals with AIV inoculation/gene methylation ratios of individuals challenged with PBS) were measured as the gene methylation fold change level. F-tests were used to compare gene methylation fold change levels in the Fayoumi and Leghorn breeds, ${ }^{*}$ indicates a significance level of $p=0.01-0.05,{ }^{* * *}$ indicates a significance level of $p<0.01$. A,B,C represented innate immune response, adaptive immune response, and oxidative stress related pathways, respectively. 


\subsection{Further Tests for Homeostasis Hypothesis in Fayoumi and Leghorn Breeds}

In Fayoumi chickens, the overall smaller changes in the difference or fold change levels in methylation or expression, with milder symptoms after AIV inoculation, supported the hypothesis of maintenance of homeostasis for disease resistance. To further test this hypothesis, we calculated the Pearson coefficient between difference levels of DMRs and difference levels of corresponding top-fold change genes with an FDR-corrected $p<0.05$. For Fayoumi chickens, a positive correlation of 0.370 was observed $(p=0.030)$, whereas a significant correlation was not found for Leghorn chickens $(\mathrm{r}=0.119$, $p=0.337$ ). This result suggested more efficient epigenetic regulation of gene expression in Fayoumi chickens compared with Leghorn chickens.

Considering that housekeeping genes are generally more stable, if the homeostasis hypothesis holds true, we expect distinct difference and fold change levels in housekeeping genes between the two breeds. As shown in Figure 3, the distribution of Fayoumi housekeeping gene expression difference was more contracted compared to the distribution in Leghorn chickens. A paired t-test was utilized to compare the mean difference in both breeds, and the housekeeping gene expression difference levels in Leghorn were significantly larger $(p=0.001)$ than those in Fayoumi, with $t=-3.2307$. F-tests were also used to compare the expression fold change levels of housekeeping genes (variance of $\log _{2}\left(\mathrm{IA} \_\mathrm{G} / \mathrm{IP} \_\mathrm{G}\right)$ ) in both breeds, and the expression fold change levels of housekeeping genes in Fayoumi were observed to be much smaller than those in Leghorn chickens, with $\mathrm{F}=1.119$ and $p=0.002$.

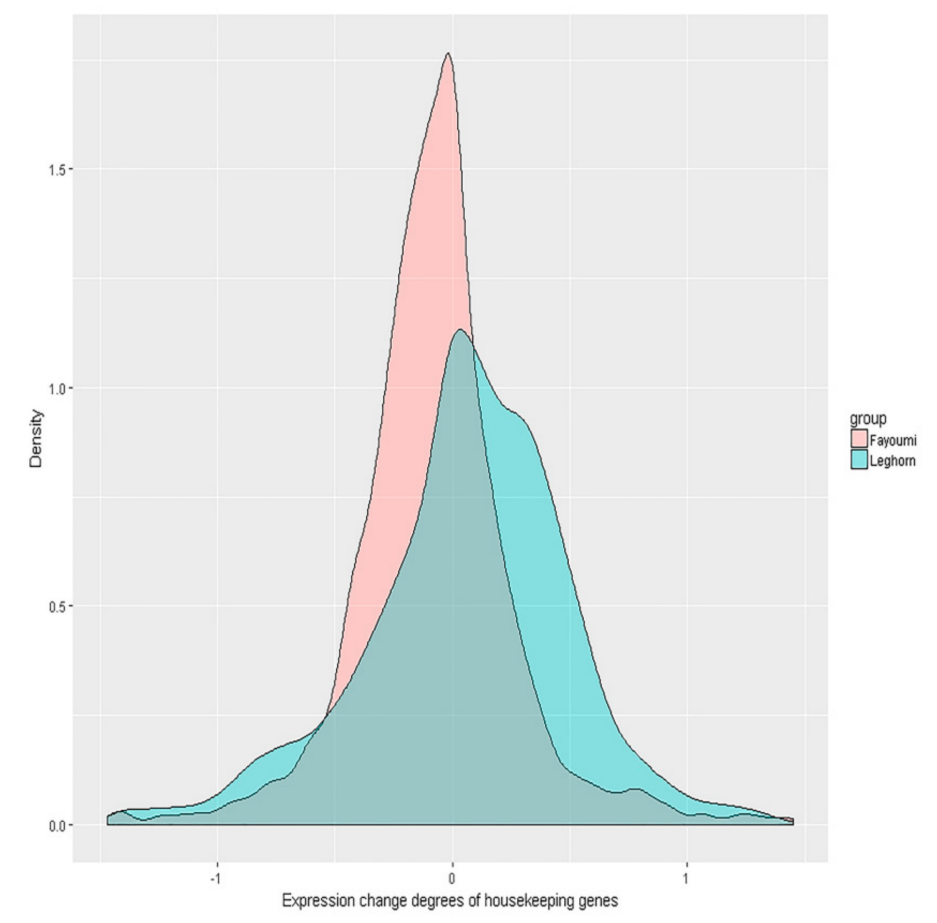

Figure 3. Housekeeping gene expression difference distribution in Fayoumi and Leghorn chickens. The $\mathrm{x}$-axis represents the housekeeping gene expression difference for the two breeds. The values were calculated as $\log _{2}$ (gene expression levels of Fayoumi individuals with AIV inoculation/gene expression levels of Fayoumi individuals challenged with PBS) or $\log _{2}$ (gene expression levels of Leghorn individuals with AIV inoculation/gene expression levels of Leghorn individuals challenged with PBS). The $y$-axis represents the density of the gene expression difference degree.

Methylation difference degrees and fold change levels were also calculated for all housekeeping genes. After averaging four replicates for each gene, we compared the methylation difference degrees of housekeeping genes in both breeds, and we found that the methylation difference degrees of Fayoumi housekeeping genes were much smaller than those of Leghorn housekeeping genes $(t=-23.870$, $p<0.0001)$. Methylation fold change levels of housekeeping genes showed statistical differences 
where Fayoumi chickens had much smaller levels than Leghorn chickens $(\mathrm{F}=1.707, p<0.0001)$, which is consistent with our hypothesis. In summary, these results suggest a distinct ability to maintain homeostasis in both breeds.

\subsection{Genetic Differentiation Between Fayoumi and Leghorn Chickens}

The Fayoumi and Leghorn chickens used in this study are two highly inbred breeds with inbreeding coefficients of over $99.9 \%[9,11,13]$. To elucidate the genetic differentiation between these two breeds at the whole genome level, we used the fixation index $\left(\mathrm{F}_{\mathrm{ST}}\right)$. By using a window size of $10 \mathrm{kbp}$ and a step size of $10 \mathrm{kbp}$, we calculated the $\mathrm{F}_{\mathrm{ST}}$ in every window and acquired its distribution for each chromosome. Consistent with our expectations, we observed that, for almost all chromosomes, the median $\mathrm{F}_{\mathrm{ST}}$ value was over 0.900, except for chromosomes 6, 7, 8, 9, 16, 25, and 28 (Table S6). Chromosome 16, where the MHC genes are located, showed a lower $\mathrm{F}_{\mathrm{ST}}$ between Leghorn and Fayoumi chickens compared to most of the other chromosomes. We specifically investigated the genomic regions harboring MHC reported by Abernathy et al. [9]. The median $\mathrm{F}_{\mathrm{ST}}$ values in the five MHC regions (195000-205000, 215000-225000, 95000-105000, 125000-135000, and 145000-155000 bp) were found to be $0.811,0.743,0.741,0.714$, and 0.707 , respectively, which were also lower than the genome average value. These results indicated that the MHC-involved process of antigen presentation might not adequately explain the distinct resistance abilities between the two breeds.

Furthermore, we calculated $\mathrm{F}_{\mathrm{ST}}$ values for all 19542 genes in the chicken genome (downloaded from the Ensemble database GRCg6a) by averaging $\mathrm{F}_{\mathrm{ST}}$ values of each base along the gene regions. The mean $\mathrm{F}_{\mathrm{ST}}$ value for all genes was 0.564 , and the $95 \%$ quantile value was 0.773 . As shown in Figure 4 , we surveyed immune response related genes by grouping them according to the order of immune response occurrence: The innate immune response, the adaptive immune response, and oxidative stress related pathways. MX1, HSP90AB1, and HSP90B1 were the only three genes that exhibited high $\mathrm{F}_{\mathrm{ST}}$ values $(0.802,0.787$, and 0.972 , respectively) in the top $5 \%$ of all genes. According to the gene annotations, MX1 is involved in the innate immune response to AIV [4,5]. On the other hand, HSP90 family genes are known to be essential for protecting against the heat shock response and providing stress tolerance [33] by buffering proteostasis against environmental stress and functioning as a hub gene regulating protein homeostasis.
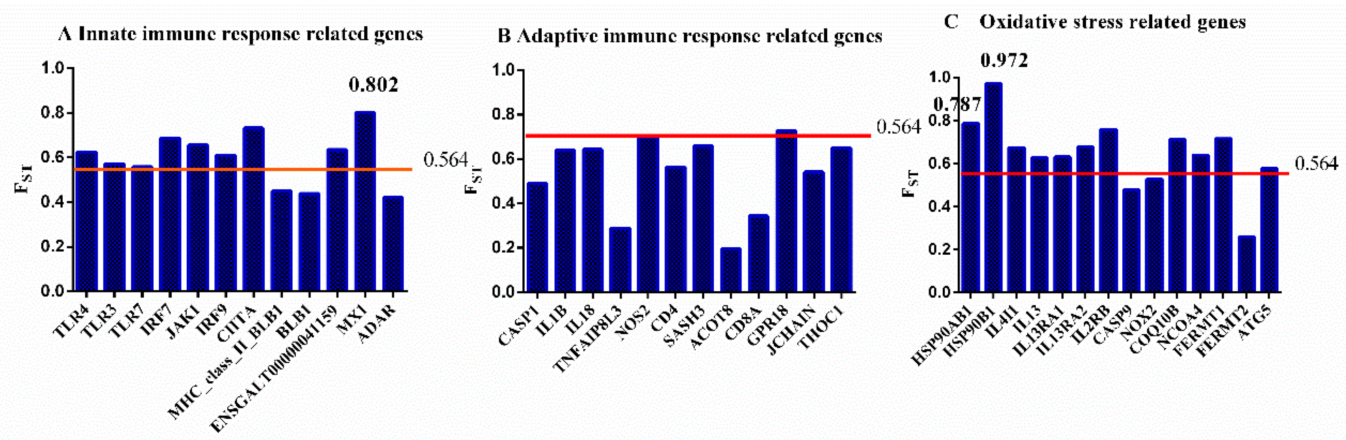

Figure 4. Fixation index $\left(\mathrm{F}_{\mathrm{ST}}\right)$ values of immune response related genes: (A) $\mathrm{F}_{\mathrm{ST}}$ values of innate immune response related genes, (B) $\mathrm{F}_{\mathrm{ST}}$ values of adaptive immune response related genes, (C) $\mathrm{F}_{\mathrm{ST}}$ values of oxidative stress related genes. The red line represents the mean $\mathrm{F}_{\mathrm{ST}}$ value of all genes in the genome.

\section{Discussion}

Highly contagious avian influenza, which spreads through the respiratory tract [37], constitutes a serious burden for both the poultry industry and public safety [1]. The rapid development of the NGS (next generation sequencing) technology has provided a fruitful opportunity to investigate the mechanisms of disease resistance of avian influenza from a broader perspective. Here, we extended our previous research [11] by studying DNA resequencing, methylation, and expression data using two 
chicken breeds with extreme AIV resistance phenotypes. Our results suggest a homeostasis hypothesis for avian influenza resistance in chickens (Figure 5).

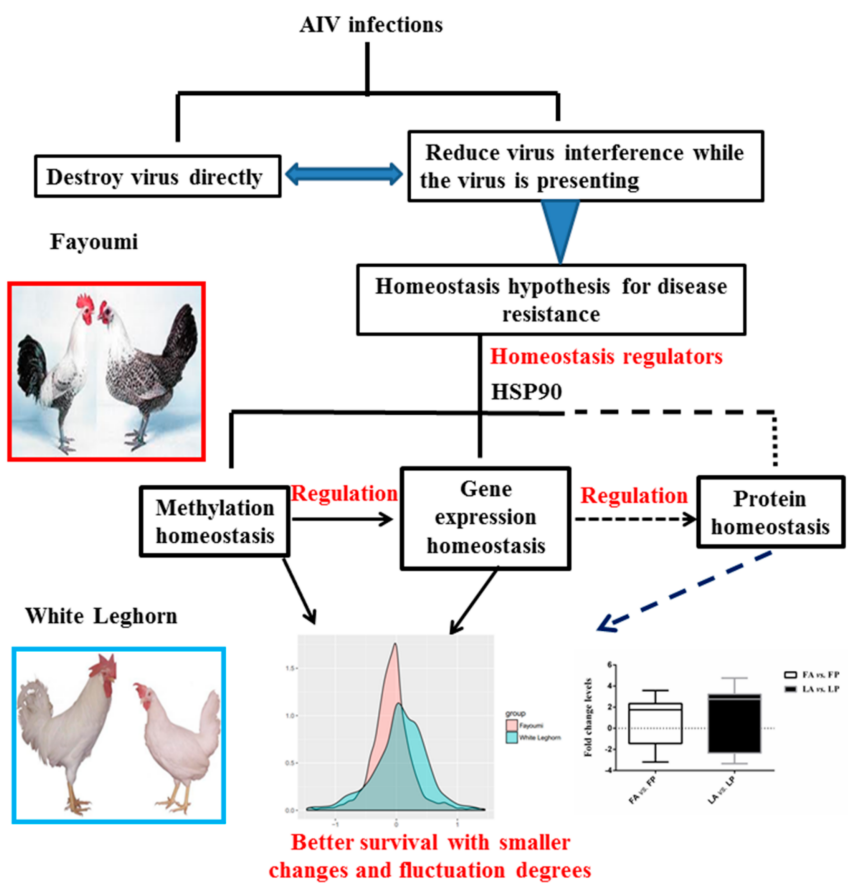

Figure 5. Scheme of the homeostasis hypothesis of avian influenza resistance in chickens. The overall homeostasis was achieved by methylation homeostasis, gene expression homeostasis, and protein homeostasis. The dashed line represents measurements that were not included in this study.

After AIV inoculation, survival requires a delicate balance between the host defense and host tolerance. The host defense is essential for the detection and elimination of foreign pathogens, while the host tolerance is vital to minimize tissue damage due to an excessive immune response. It was found that when challenged with the RNA virus, the host prevented overproduction of type I IFNs (interferons) to reduce host tissue damage, which validated the viewpoint at the molecular level [38]. It was also reported that the change in mean corresponded to the plasticity of the phenotype, whereas the change in variance reflected sensitivity to noise in tested environments [39]. In Fayoumi, we found smaller degrees in both the difference and fold change levels for both methylation as a sensor and regulator of the changes in the surrounding environment and gene expression as a functional response to the changes in the surrounding environment, even for housekeeping genes (Figure 5). We also observed that Fayoumi exhibited a clearer relationship between changes in methylation and gene expression compared to Leghorn chickens, indicating a more ordered or successful regulation or information transmission. Together with the observation of comparable virus titers in two infected breeds, this suggests a homeostasis hypothesis of avian influenza resistance in chickens as an indirect anti-virus mechanism, in addition to direct anti-virus mechanisms of destroying or blocking the entry of the virus into the cell (Figure 5).

To validate that the obtained top-fold change genes were biologically meaningful, we analyzed the proteomics data of the sample samples (data not published). We found that most of the top-fold change genes with FDR-corrected $p<0.05$ also showed significant differences at the protein level. Among the $85(35+50)$ top-fold change genes, 17 transcripts could not be translated into proteins, and 12 top-fold change genes were labeled as uncharacterized proteins. We found that 18 top-fold change genes exhibited a significant difference in FA compared to FP and LA compared to LP. In order to clarify the change trends of top-fold change genes at the RNA and protein levels, we drew a heatmap with expression values measured as $\log _{2}$ (expression values at the corresponding level of individuals 
with AIV inoculation/expression values of individuals challenged with PBS) (Figure S4). As shown in Figure S4, top-fold change genes obtained at the RNA level also showed the same regulation trend in the same comparison group.

Our homeostasis hypothesis for disease resistance in chickens can be regarded as an extension of the canalization hypothesis in the area of disease resistance. The canalization hypothesis predicted an invariable phenotype regardless of the environment variability [39]. In the process of AIV resistance, we suggest that genetics and epigenetics work together, contributing to the resistance phenotype. At the genetic differentiation level, we detected MX1, HSP90AB1, and HSP90B1, whose F $\mathrm{ST}_{\mathrm{ST}}$ vaes passed the whole genome top $5 \%$ threshold. It is well known that non-synonymous codon changes could impact gene function. To identify functional sites within MX1, HSP90AB1, and HSP90B1, we compared $\mathrm{dn} / \mathrm{ds}$ for these three genes. MX1 is composed of 705 amino acids, and we detected that the $605 \mathrm{th}, 317 \mathrm{th}, 324 \mathrm{th}$, and 354th amino acids were under positive selection with $p$-values of 0.03 , $0.05,0.05$, and 0.05 , respectively. The most interesting site in the MX1 cDNA was Ser (S) to Asn (N) substitution at the amino acid position 631 (S631N) caused by mutation G2032A. However, whether this site contributes to AIV resistance is controversial. Previously, Wang et al. experimentally validated that the MX1 amino acid 631 is not relevant to AIV replication in chickens both in ovo and in vivo [5]. In our analysis, the amino acid site 631 in $M X 1$ was not shown to be positively selected either. We applied the same method for HSP90AB1 and HSP90B1; unfortunately, no significant site was found in HSP90AB1 or HSP90B1 with a $p$-value threshold of 0.1 .

In our results, the MX1 protein was also shown to differ significantly for FA compared to FP and LA compared to LP in the lung tissues (Figure S5). After AIV inoculation, the Fayoumi breed upregulated the MX1 protein, whereas Leghorn chickens did not produce the MX1 protein (Figure S5A). For the HSP90AB1 protein, in the Fayoumi breed, the fold change value of FA compared to FP was 5.1146 with a $p$-value of $2.3965 \times 10^{-111}$. However, there was no significant difference for LA compared to LP (Figure S5B). For the HSP90B1 protein, both breeds exhibited a significant difference after virus inoculation; however, Fayoumi upregulated HSP90B1 to a greater extent with a fold change of 42.3152, whereas the fold change of Leghorn chickens was 2.2874 (Figure S5C). The MX1 gene has been systematically studied in human and mice anti influenza virus infections. The MX1 gene is a dynamin-like guanosine triphosphatase that blocks the importation of influenza ribonucleoprotein, a process known to be sufficient to confer resistance to influenza viruses [40,41]. In humans, the MX1 gene product MxA inhibits a step of the influenza virus replication cycle following primary transcription [42]. Genetically modified $M X 1+/+$ mice showed more resistance to the influenza virus inoculation than MX1-deficient mice [43-45]. In our study, Fayoumi chickens showed a higher MX1 protein level after AIV inoculation, whereas expression of the MX1 protein remained at a relatively low level in Leghorn chickens both before and after inoculation. Our results suggested that the MX1 gene may play an important role in the difference in AIV resistance between Fayoumi and Leghorn chickens.

The HSP90 gene family is one of the best studied examples of a general capacitor that buffers both genetic and environmental perturbations [46]. In our result, we found that HSP90 showed high genetic differentiation level between the two breeds. In addition, the degrees of change and fold change levels in HSP90 in terms of methylation and gene expression were larger in Leghorn compared to Fayoumi chickens, which indicates higher robustness to AIV perturbations in Fayoumi, which is in line with the canalization hypothesis.

The key genes HSP90AB1 and HSP90B1 were reported to function in innate immunity, and we showed that these two HSP90 family genes also act as homeostasis markers in AIV infections. The HSP90AB1 gene is one of the major two cytosolic isoforms of the HSP90 family [47]. The HSP90 gene family is relevant to numerous cellular pathways and is known to play roles in protein stabilization and the adaptive response to stress [48]. Wahl, A.; et al. found that approximately 30\% of host changed proteins mediated by influenza directly interact with HSP90AB1 [49]. By complexing with AKT, HSP90AB1 activates downstream antiapoptotic NF-90FD5B20BF, which is an important aspect of cardiac cellular defense strategies [50]. By directly associating with HMGB1, HSP90B1 plays an 
important role in the process of cellular and immune homeostasis at the airway mucosal surface [51]. Under this viewpoint, it is conceivable that increasing HSP90AB1 and HSP90B1 expression levels might efficiently reduce damage from AIV infections. For example, reactivating HSP90AB1 expression by genetic modification or developing drugs to stimulate HSP90AB1 expression might provide new opportunities for AIV resistance. In summary, evidence for genetic differentiation, methylation, and gene expression supports the hypothesis of maintaining homeostasis in disease resistance. Fayoumi chickens possess a superior ability to maintain homeostasis compared to Leghorn chickens, which could constitute another form of evolutionary robustness. However, further work is required to discover the specific gene functions that contribute to homeostasis in disease resistance in detail.

The avian influenza virus is one of the zoonotic pathogens that can cross the species barrier and result in epidemic outbreaks, which is in common with viruses in bats that can make the jump to humans. So far, multiple scholars have reported that bats remain disease-free when infected with the highly pathogenic RNA viruses they carry and bats can coexist with them without detectable fitness costs using measures such as overt signs of inflammation [52]. This is in line with findings from our results, which found that Fayoumi was more robust than Leghorn lines challenged with AIV. Based on the comparison of 10 bat genomes sequenced so far and mamal genomes, researchers found that PYHIN genes were entirely lost [53], which could interact with stimulator of interferon genes (STING) and activate the inflammasome. It has been hypothesized that the absence of the PYHIN family may allow bats to limit activation of the innate immune response to damaged self-DNA generated by RNA viral infection, thus avoiding excessive inflammation [53,54]. Zhou et al. found that if an antiviral immune pathway STING-interferon pathway in bats is dampened that bats can maintain just enough defenses against illness without triggering the immune systems from going into overdrive [55]. However, in humans and other mammals, an immune-based over-response to one of these and other pathogenic viruses can trigger severe illness. Dublin researchers have also shown that compared to the immune response of a mouse, bat macrophages can rapidly mount a robust antiviral response whenever a pathogen is detected, and, bat immune system can quickly reverse their response by releasing anti-inflammatory cytokines [56]. Moreover, bat MX1 significantly reduced the polymerase activity of viruses circulating in bats, including Ebola and influenza A-like viruses [57]. By increasing pathogen replication control and mitigating any immunopathology through decreased inflammatory responses, bat increased disease tolerance.

Supplementary Materials: The following are available online at http://www.mdpi.com/2073-4425/10/7/543/s1, Figure S1: Pathway annotation of differentially expressed genes with FDR-corrected $p<0.05$ for Fayoumi with avian influenza virus (AIV) inoculation compared to Fayoumi challenged with PBS, Figure S2: Pathway annotation of differentially expressed genes with FDR-corrected $p<0.05$ for Leghorn chickens with AIV inoculation compared to Leghorn chickens challenged with PBS, Figure S3: DNA methylation level along the gene structure in all samples, Figure S4: Heatmap of top-fold change genes with FDR-corrected $p<0.05$ at the RNA and protein levels, Figure S5: Protein regulation trends of MX1, HSP90AB1, and HSP90B1 between Fayoumi and Leghorn breeds. Table S1: Gene expression difference and fold change levels in immune response related genes, Table S2: Whole genome bisulfite sequencing (WGBS) data statistics summary, Table S3: Differentially methylated sites and regions among groups, Table S4: Pathway annotation of genes located within differentially methylated regions (DMRs) in FA compared to FP and LA compared to LP, Table S5: T-test comparison of gene methylation difference in immune response related genes for Fayoumi and Leghorn chickens, Table S6: Fixation index $\left(\mathrm{F}_{\mathrm{ST}}\right)$ statistics summary for each chromosome.

Author Contributions: Conceptualization, Y.Z.; Funding acquisition, X.H. and Y.Z.; Investigation, J.A., J.L., Y.W., J.W. and Q.L.; Project administration, N.L.; Resources, H.Z. and X.H.; Validation, J.A., J.L., Y.W. and Q.L.; Visualization, J.A. and J.W.; Writing—original draft, J.A.; Writing-review and editing, Y.Z.

Funding: This work was financially supported by the National Natural Science Foundation of China (U1704233), and the National High Technology Research and Development Program of China (no.2013AA102501).

Acknowledgments: The authors are grateful to the anonymous reviewers for their insightful comments and suggestions. We thank the support of the high-performance computing platform of the State Key Laboratory of Agrobiotechnology.

Conflicts of Interest: The authors declare no competing financial interests. 


\section{References}

1. Dhama, K.; Chakraborty, S.; Tiwari, R.; Kumar, A.; Rahal, A.; Latheef, K.S.; Wani, M.Y.; Kapoor, S. Avian/Bird Flu Virus: Poultry Pathogen Having Zoonotic and Pandemic Threats: A Review. J. Med. Sci. 2013, 13, 301-315. [CrossRef]

2. Francisco, N.; Donadel, M.; Jit, M.; Hutubessy, R. A systematic review of the social and economic burden of influenza in low- and middle-income countries. Vaccine 2015, 33, 6537-6544. [CrossRef]

3. Suarez, D.L.; Schultz-Cherry, S. Immunology of avian influenza virus: A review. Dev. Comp. Immunol. 2000, 24, 269-283. [CrossRef]

4. $\quad$ Ewald, S.J.; Kapczynski, D.R.; Livant, E.J.; Suarez, D.L.; Ralph, J.; McLeod, S.; Miller, C. Association of Mx1 Asn631 variant alleles with reductions in morbidity, early mortality, viral shedding, and cytokine responses in chickens infected with a highly pathogenic avian influenza virus. Immunogenetics 2011, 63, 363-375. [CrossRef] [PubMed]

5. Wang, Y.; Brahmakshatriya, V.; Lupiani, B.; Reddy, S.; Okimoto, R.; Li, X.; Chiang, H.; Zhou, H. Associations of chicken Mx1 polymorphism with antiviral responses in avian influenza virus infected embryos and broilers. Poult. Sci. 2012, 91, 3019-3024. [CrossRef] [PubMed]

6. Lyall, J.; Irvine, R.M.; Sherman, A.; McKinley, T.J.; Núñez, A.; Purdie, A.; Outtrim, L.; Brown, I.H.; Rolleston, S.G.; Sang, H.; et al. Suppression of avian influenza transmission in genetically modified chickens. Science 2011, 331, 223-226. [CrossRef] [PubMed]

7. June, B.S.; Yuk, S.; Jang, Y.; Choi, H.; Jeon, M.; Erdene-Ochir, T.O.; Kwon, J.; Noh, J.; Sun, K.J.; Gyu, Y.J.; et al. Transgenic Chickens Expressing the 3D8 Single Chain Variable Fragment Protein Suppress Avian Influenza Transmission. Sci. Rep. 2017, 7, 1-11. [CrossRef]

8. Ledur, M.C.; Fairfull, R.W.; McMillan, I.; Asseltine, L. Genetic effects of aging on egg production traits in the first laying cycle of wWhite Leghorn strains and strain crosses. Poult. Sci. 2000, 3, 296-304. [CrossRef]

9. Abernathy, J.; Li, X.; Jia, X.; Chou, W.; Lamont, S.J.; Crooijmans, R.; Zhou, H. Copy number variation in Fayoumi and Leghorn chickens analyzed using array comparative genomic hybridization. Anim. Genet. 2014, 45, 400-411. [CrossRef]

10. Marie-Hélène, P.L.; Bertrand, B.; Jean-Luc, C.; Frédérique, P.; Katia, F.; Leroux, S.; Hélène, L.; Aurélie, T.; David, G.; Jean-Michel, R.; et al. Microsatellite mapping of QTLs affecting resistance to coccidiosis (Eimeria tenella) in a Fayoumi $\times$ White Leghorn cross. BMC Genom. 2009, 10, 31-44. [CrossRef]

11. Li, J.; Li, R.; Wang, Y.; Hu, X.; Zhao, Y.; Li, L.; Feng, C.; Gu, X.; Liang, F.; Lamont, S.J.; et al. Genome-wide DNA methylome variation in two genetically distinct chicken lines using MethylC-seq. BMC Genom. 2015, 16, 1-13. [CrossRef] [PubMed]

12. Wang, Y.; Lupiani, B.; Reddy, S.M.; Lamont, S.J.; Zhou, H. RNA-seq analysis revealed novel genes and signaling pathway associated with disease resistance to avian influenza virus infection in chickens. Poult. Sci. 2014, 93, 485-493. [CrossRef] [PubMed]

13. Fleming, D.S.; Koltes, J.E.; Fritz-Waters, E.R.; Rothschild, M.F.; Schmidt, C.J.; Ashwell, C.M.; Persia, M.E.; Reecy, J.M.; Lamont, S.J. Single nucleotide variant discovery of highly inbred Leghorn and Fayoumi chicken breeds using pooled whole genome resequencing data reveals insights into phenotype differences. BMC Genom. 2016, 17, 812. [CrossRef] [PubMed]

14. Ramsay, D.S.; Woods, S.C. Clarifying the roles of homeostasis and allostasis in physiological regulation. Psychol. Rev. 2014, 121, 25-247. [CrossRef] [PubMed]

15. Pacis, A.; Tailleux, L.; Morin, A.M.; Lambourne, J.; MacIsaac, J.L.; Yotova, V.; Dumaine, A.; Danckaert, A.; Luca, F.; Grenier, J.; et al. Bacterial infection remodels the DNA methylation landscape of human dendritic cells. Genome Res. 2015, 25, 1801-1811. [CrossRef] [PubMed]

16. Shang, X.; Su, J.; Wan, Q.; Su, J.; Feng, X. CpG methylation in the 5'-flanking region of LGP2 gene lacks association with resistance/susceptibility to GCRV but contributes to the differential expression between muscle and spleen tissues in grass carp, Ctenopharyngodon idella. Fish Shellfish Immunol. 2014, 40, 154-163. [CrossRef] [PubMed]

17. Lai, A.Y.; Mav, D.; Shah, R.; Grimm, S.A.; Phadke, D.; Hatzi, K.; Melnick, A.; Geigerman, C.; Sobol, S.E.; Jaye, D.L.; et al. DNA methylation profiling in human B cells reveals immune regulatory elements and epigenetic plasticity at Alu elements during B-cell activation. Genome Res. 2013, 23, 2030-2041. [CrossRef] 
18. Xu, H.; Zhu, X.; Hu, Y.; Li, Z.; Zhang, X.; Nie, Q.; Nolan, L.K.; Lamont, S.J. DNA methylome in spleen of avian pathogenic escherichia coli-challenged broilers and integration with mRNA expression. Sci. Rep. 2015, 4, 8303. [CrossRef]

19. Yu, A.; Lepere, G.; Jay, F.; Wang, J.; Bapaume, L.; Wang, Y.; Abraham, A.L.; Penterman, J.; Fischer, R.L.; Voinnet, O.; et al. Dynamics and biological relevance of DNA demethylation in Arabidopsis antibacterial defense. Proc. Natl. Acad. Sci. USA 2013, 110, 2389-2394. [CrossRef]

20. Xu, S.; Yao, H.; Zhang, J.; Zhang, Z.; Wang, J.; Zhang, J.; Jiang, Z. The oxidative damage and disbalance of calcium homeostasis in brain of chicken induced by selenium deficiency. Biol. Trace Elem. Res. 2013, 151, 225-233. [CrossRef]

21. Waddington, C.H. Canalization of development and the inheritance of acquired characters. Nature 1942, 150, 563-565. [CrossRef]

22. Takahashi, K.H. Multiple modes of canalization: Links between genetic, environmental canalizations and developmental stability, and their trait-specificity. Semin. Cell Dev. Biol. 2019, 88, 14-20. [CrossRef] [PubMed]

23. Trapnell, C.; Roberts, A.; Goff, L.; Pertea, G.; Kim, D. Differential gene and transcript expression analysis of RNA-seq experiments with TopHat and Cufflinks. Nat. Protoc. 2012, 7, 562-578. [CrossRef] [PubMed]

24. Krueger, F.; Andrews, S.R. Bismark: A flexible aligner and methylation caller for Bisulfite-Seq applications. Bioinformatics 2011, 27, 1571-1572. [CrossRef] [PubMed]

25. Dolzhenko, E.; Smith, A.D. Using beta-binomial regression for high-precision differential methylation analysis in multifactor whole-genome bisulfite sequencing experiments. BMC Bioinform. 2014, 15, 210-215. [CrossRef] [PubMed]

26. Li, H.; Durbin, R. Fast and accurate short read alignment with Burrows-Wheeler transform. Bioinformatics 2009, 25, 1754-1760. [CrossRef]

27. McKenna, A.; Hanna, M.; Banks, E.; Sivachenko, A.; Cibulskis, K.; Kernytsky, A.; Garimella, K.; Altshuler, D.; Gabriel, S.; Daly, M.; et al. The Genome Analysis Toolkit: A MapReduce framework for analyzing next-generation DNA sequencing data. Genome Res. 2010, 20, 1297-1303. [CrossRef]

28. Weir, B.S.; Cockerham, C.C. Estimating F-statistics for the analysis of population structure. Evolution 1984, 38, 1358-1370. [CrossRef]

29. Quinlan, A.R.; Hall, I.M. BEDTools: A flexible suite of utilities for comparing genomic features. Bioinformatics 2010, 26, 841-842. [CrossRef]

30. Pond, S.L.; Frost, S.D.; Muse, S.V. HyPhy: Hypothesis testing using phylogenies. Binformatics 2005, 21, 676-679. [CrossRef]

31. Weaver, S.; Shank, S.D.; Spielman, S.J.; Li, M.; Muse, S.V.; Kosakovsky Pond, S.L. Datamonkey 2.0: A modern web application for characterizing selective and other evolutionary processes. Mol. Biol. Evol. 2018, 35, 773-777. [CrossRef] [PubMed]

32. Murrell, B.; Wertheim, J.O.; Moola, S.; Weighill, T.; Scheffler, K.; Kosakovsky, P.S.L. Detecting individual sites subject to episodic diversifying selection. PLoS Genet. 2012, 8, e1002764. [CrossRef] [PubMed]

33. Mikko, T.; Daniel, F.J.; Lindquist, S. HSP90 at the hub of protein homeostasis: Emerging mechanistic insights. Nat. Rev. Mol. Cell Biol. 2010, 11, 515-528. [CrossRef]

34. Suzuki, M.M.; Bird, A. DNA methylation landscapes: Provocative insights from epigenomics. Nat. Rev. Genet. 2008, 9, 465-476. [CrossRef] [PubMed]

35. Jones, P.A. Functions of DNA methylation: Islands, start sites, gene bodies and beyond. Nat. Rev. Genet. 2012, 13, 484-492. [CrossRef]

36. Maunakea, K.A.; Chepelev, I.; Cui, K.; Zhao, K. Intragenic DNA methylation modulates alternative splicing by recruiting MeCP2 to promote exon recognition. Cell Res. 2013, 23, 1256-1269. [CrossRef] [PubMed]

37. Costa, T.; Chaves, A.J.; Valle, R.; Darji, A.; van Riel, D.; Kuiken, T.; Majo, N.; Ramis, A. Distribution patterns of influenza virus receptors and viral attachment patterns in the respiratory and intestinal tracts of seven avian species. Vet. Res. 2012, 43,1-13. [CrossRef]

38. Jiang, M.; Zhang, S.; Yang, Z.; Lin, H.; Zhu, J.; Liu, L.; Wang, W.; Liu, S.; Liu, W.; Ma, Y.; et al. Self-recognition of an inducible host lncRNA by RIG-I feedback restricts innate immune response. Cell 2018, 173, 906-919. [CrossRef]

39. Félix, M.; Barkoulas, M. Pervasive robustness in biological systems. Nat. Rev. Genet. 2015, 16, $483-496$. [CrossRef] 
40. Phillips, A.M.; Ponomarenko, A.I.; Chen, K.; Ashenberg, O.; Miao, J.; McHugh, S.M.; Butty, V.L.; Whittaker, C.A.; Moore, C.L.; Bloom, J.D.; et al. Destabilized adaptive influenza variants critical for innate immune system escape are potentiated by host chaperones. PLoS Biol. 2018, 16, e3000008. [CrossRef]

41. Tumpey, T.M.; Szretter, K.J.; Van Hoeven, N.; Katz, J.M.; Kochs, G.; Haller, O.; Garcia-Sastre, A.; Staeheli, P. The Mx1 gene protects mice against the pandemic 1918 and highly lethal human H5N1 influenza viruses. J. Virol. 2007, 81, 10818-10821. [CrossRef] [PubMed]

42. Pavlovic, J.; Haller, O.; Staeheli, P. Human and mouse Mx proteins inhibit different steps of the influenza virus multiplication cycle. J. Virol. 1992, 66, 2564-2569. [PubMed]

43. Pillai, P.S.; Molony, R.D.; Martinod, K.; Dong, H.; Pang, I.K.; Tal, M.C.; Solis, A.G.; Bielecki, P.; Mohanty, S.; Trentalange, M.; et al. Mx1 reveals innate pathways to antiviral resistance and lethal influenza disease. Science 2016, 352, 463-466. [CrossRef] [PubMed]

44. Kaminski, M.M.; Ohnemus, A.; Cornitescu, M.; Staeheli, P. Plasmacytoid dendritic cells and Toll-like receptor 7-dependent signalling promote efficient protection of mice against highly virulent influenza A virus. J. Gen. Virol. 2012, 93, 555-559. [CrossRef] [PubMed]

45. Grimm, D.; Staeheli, P.; Hufbauer, M.; Koerner, I.; Martinez-Sobrido, L.; Solorzano, A.; Garcia-Sastre, A.; Haller, O.; Kochs, G. Replication fitness determines high virulence of influenza A virus in mice carrying functional Mx1 resistance gene. Proc. Natl. Acad. Sci. USA 2007, 104, 6806-6811. [CrossRef] [PubMed]

46. Takahashi, K.H. Novel genetic capacitors and potentiators for the natural genetic variation of sensory bristles and their trait specificity inDrosophila melanogaster. Mol. Ecol. 2015, 24, 5561-5572. [CrossRef] [PubMed]

47. Chen, B.; Piel, W.H.; Gui, L.; Bruford, E.; Monteiro, A. The HSP90 family of genes in the human genome: Insights into their divergence and evolution. Genomics 2005, 86, 627-637. [CrossRef] [PubMed]

48. Makhnevych, T.; Houry, W.A. The role of Hsp90 in protein complex assembly. Biochim. Biophys. Acta 2012, 1823, 674-682. [CrossRef] [PubMed]

49. Wahl, A.; Schafer, F.; Bardet, W.; Hildebrand, W.H. HLA class I molecules reflect an altered host proteome after influenza virus infection. Hum. Immunol. 2010, 71, 14-22. [CrossRef] [PubMed]

50. Grabek, K.R.; Karimpour-Fard, A.; Epperson, L.E.; Hindle, A.; Hunter, L.E.; Martin, S.L. Multistate proteomics analysis reveals novel strategies used by a hibernator to precondition the heart and conserve ATP for winter heterothermy. Physiol. Genom. 2011, 43, 1263-1275. [CrossRef] [PubMed]

51. Wong, S.L.; To, J.; Santos, J.; Allam, V.S.R.R.; Dalton, J.P.; Djordjevic, S.P.; Donnelly, S.; Padula, M.P.; Sukkar, M.B. Proteomic analysis of extracellular HMGB1 identifies binding partners and exposes its potential role in airway epithelial cell homeostasis. J. Proteome Res. 2018, 17, 33-45. [CrossRef] [PubMed]

52. Baker, M.L.; Schountz, T.; Wang, L.F. Antiviral immune responses of bats: A review. Zoonoses Public Health 2013, 60, 104-116. [CrossRef] [PubMed]

53. Ahn, M.; Cui, J.; Irving, A.T.; Wang, L. Unique loss of the PYHIN gene family in bats amongst mammals: Implications for inflammasome sensing. Sci. Rep. 2016, 6, 21722. [CrossRef] [PubMed]

54. Li, N.; Parrish, M.; Chan, T.K.; Yin, L.; Rai, P.; Yoshiyuki, Y.; Abolhassani, N.; Tan, K.B.; Kiraly, O.; Chow, V.T.K.; et al. Influenza infection induces host DNA damage and dynamic DNA damage responses during tissue regeneration. Cell Mol. Life Sci. 2015, 72, 2973-2988. [CrossRef] [PubMed]

55. Xie, J.; Li, Y.; Shen, X.; Goh, G.; Zhu, Y.; Cui, J.; Wang, L.; Shi, Z.; Zhou, P. Dampened STING-dependent interferon activation in bats. Cell Host Microbe 2018, 23, 297-301. [CrossRef] [PubMed]

56. Joanna, K.; Hughes, G.M.; Palsson-McDermott, E.M.; Quinn, S.R.; Puechmaille, J.S.; O'Neill, L.A.J.; Teeling, E.C. A potent anti-inflammatory response in bat macrophages may be linked to extended longevity and viral tolerance. Acta Chiropterol. 2017, 2, 219-228. [CrossRef]

57. Fuchs, J.; Hölzer, M.; Schilling, M.; Patzina, C.; Andreas, S.; Hoenen, T.; Zimmer, G.; Marz, M.; Weber, F.; Müller, M.A. Evolution and antiviral specificity of interferon-induced Mx proteins of bats against Ebola-, Influenza-, and other RNA viruses. J. Virol. 2017, 91, e317-e361. [CrossRef]

(C) 2019 by the authors. Licensee MDPI, Basel, Switzerland. This article is an open access article distributed under the terms and conditions of the Creative Commons Attribution (CC BY) license (http://creativecommons.org/licenses/by/4.0/). 